\title{
Energy Efficiency Analysis in Relay Assisted Hybrid-ARQ Communications
}

\author{
Mohamad Maaz
}

Jeudi de la com'

20th September 2012 


\section{Outline}

(1) Introduction

(2) Motivations and system model

(3) Related works and contributions

(4) Theoretical energy efficiency in relay assisted network

(5) Simulation results

6) Conclusions and future works 


\section{Introduction}

\section{Relay assisted cellular networks}

Intermediate relays between the base station (BS) and users leads to several gains:

- Coverage enhancement

- Higher data rates

- High cell capacity (number of users)

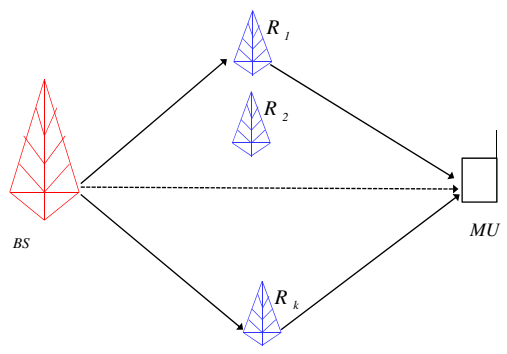




\section{Motivations and system model}

- Aim: Energy efficiency analysis in relay assisted HARQ schemes

$$
\eta_{E}=\frac{(1-P E R) \cdot N_{b}}{\bar{E}_{p k t}} \quad(\text { bits } / \text { joule })
$$

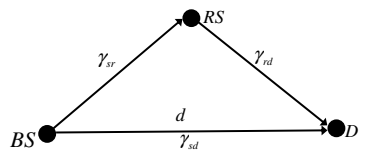

\section{System protocol}

- BS broadcasts the packet to RS and D

- If $\mathrm{RS}$ has received the packet and D not yet, $\mathrm{RS}$ starts retransmitting the packet

- Each receiving node combines the retransmitted packet with the previous ones by means of Chase combining

- The number of retransmissions is limited to $N_{\max }$ 


\section{Related works and contributions}

\section{Related works}

- In [1], the authors studied the energy efficiency for cooperative and non cooperative HARQ schemes for users constrained in outage probability. In contrast, our analysis takes into account the PER

- In [2], the authors studied the end-to-end throughput and the energy efficiency of opportunistic and multi-hop routing protocols without considering the Chase combining technique

- In [3], the throughput of HARQ-CC protocol with cooperation has been analyzed. However, this analysis is complex in comparison to our analysis

\section{Contributions}

- New simple analytical model of PER in relay-assisted HARQ-CC schemes

- Energy efficiency analysis in HARQ-CC for relay-assisted schemes with direct link

[1]- I. Stanojev, O. Simeone, Y. Bar-Ness, and K. Dong Ho, "Energy efficiency of non-collaborative and collaborative hybrid-ARQ protocols," IEEE Transactions on Wireless Communications, vol. 8, pp. 326-335, Jan. 2009.

[2]- D. Chiarotto, O. Simeone, and M. Zorzi, "Throughput and Energy Efficiency of Opportunistic Routing with Type-I HARQ in Linear Multihop Networks, "in IEEE Global Telecommunications Conference (GLOBECOM 2010), pp. 1-6, Dec. 2010.

[3]- X. Lagrange, "Performance of Chase-Combining HARQ protocol with Cooperation," tech. rep., TRITA - ICT - COS - 1006 ISSN 1653 - 6347 ISRN $\mathrm{KHT} / \mathrm{COS} / \mathrm{R}-10 / 06-\mathrm{SE}, 2010$. 


\section{Delay theoretical model}

- The average delay in cooperative scheme is:

$$
\bar{N}_{t_{\text {coop }}}=\bar{N}_{t_{s}}+\bar{N}_{t_{r}}
$$

- The average delay when the source is transmitting:

$$
\begin{aligned}
\bar{N}_{t_{s}}= & \sum_{n=1}^{N_{\max }-1} n\left(Q_{s d}(n) P_{s r}(n-1)+P_{s d}(n) Q_{s r}(n)\right)+N_{\max }\left(Q_{s d}\left(N_{\max }\right) P_{s r}\left(N_{\max }-1\right)\right) \\
& +N_{\max }\left(P_{s r}\left(N_{\max }-1\right) P_{s d}\left(N_{\max }\right)\right)
\end{aligned}
$$

where $Q_{s d}(n)=P_{s d}(n-1)-P_{s d}(n)$ and $Q_{s r}(n)=P_{s r}(n-1)-P_{s r}(n)$.

- $\bar{N}_{t_{r}}$ can be expressed as:

$$
\bar{N}_{t_{r}}=\sum_{n^{\prime}=1}^{N_{\max }-1} Q_{s r}\left(n^{\prime}\right)\left(\sum_{n=n^{\prime}+1}^{N_{\max }}\left(n-n^{\prime}\right) Q_{r d}\left(n, n^{\prime}\right)+\left(N_{\max }-n^{\prime}\right) \cdot P_{r d}\left(N_{\max }, n^{\prime}\right)\right)
$$




\section{Probability of error (1)}

- The packet error rate using HARQ-CC is defined as follows:

$$
P E R_{\text {coop }}=1-\left(\sum_{n=1}^{N_{\max }} Q_{s}(n)+\sum_{n^{\prime}=1}^{N_{\max }-1} \sum_{n=n^{\prime}+1}^{N_{\max }} Q_{r}\left(n, n^{\prime}\right)\right)
$$

- $Q_{r}\left(n, n^{\prime}\right)$ is as follows:

$$
Q_{r}\left(n, n^{\prime}\right)=Q_{s r}\left(n^{\prime}\right)\left(P_{r d}\left(n-1, n^{\prime}\right)-P_{r d}\left(n, n^{\prime}\right)\right)
$$

- $Q_{s}(n)$ is calculated as:

$$
Q_{s}(n)=P_{s r}(n-1)\left(P_{s d}(n-1)-P_{s d}(n)\right)
$$

- The error probability $P(n)$ can be calculated by:

$$
\begin{aligned}
P(n) & =\int_{0}^{\infty} \int_{0}^{\infty} \ldots \int_{0}^{\infty} f\left(\gamma_{1}\right) f\left(\gamma_{1}+\gamma_{2}\right) \cdots f\left(\gamma_{1}+\gamma_{2}+\ldots \gamma_{n}\right) \\
& \times p_{\gamma_{s d}}\left(\gamma_{1}\right) \cdot p_{\gamma_{s d}}\left(\gamma_{2}\right) \cdots p_{\gamma_{s d}}\left(\gamma_{n}\right) d \gamma_{1} d \gamma_{2} \ldots d \gamma_{n}
\end{aligned}
$$

- Equation (7) can be approximated by:

$$
\begin{aligned}
P(n) & \approx \underbrace{\int_{0}^{\infty} \int_{0}^{\infty} \cdots \int_{0}^{\infty}}_{n \text { folds }} f\left(\gamma_{1}+\gamma_{2}+\ldots \gamma_{n}\right) \\
& \times p_{\gamma_{s d}}\left(\gamma_{1}\right) \cdot p_{\gamma_{s d}}\left(\gamma_{2}\right) \cdots p_{\gamma_{s d}}\left(\gamma_{n}\right) d \gamma_{1} d \gamma_{2} \ldots d \gamma_{n}
\end{aligned}
$$




\section{Probability of error (2)}

- The packet error rate $f(\gamma)$ can be fitted by:

$$
f(\gamma)=\left\{\begin{array}{cc}
a \cdot \exp (-g \gamma) & \text { if } \gamma \geq \gamma_{M} \\
1 & \text { otherwise }
\end{array}\right.
$$

where $a, g$ and $\gamma_{M}$ depend on the packet length, modulation and coding schemes.

- $P(n)$ can be split into three parts as [4]:

$$
P(n)=A_{n}+\sum_{m=1}^{n-1} B_{n, m}+C_{n}
$$

where $A_{n}, B_{n, m}$ and $C_{n}$ can be proved to be:

$$
\begin{gathered}
A_{n}=1-\exp \left(-\frac{\gamma_{M}}{\bar{\gamma}_{s d}}\right) \sum_{k=0}^{n-1} \frac{1}{k !}\left(\frac{\gamma_{M}}{\bar{\gamma}_{s d}}\right)^{k} \\
B_{n, m}=\left(\frac{\gamma_{M}^{m}}{m !}\right)\left[\frac{1}{1+g \bar{\gamma}_{s d}}\right]^{n-m}\left(\frac{1}{\bar{\gamma}_{s d}}\right)^{m} \exp \left(-\frac{\gamma_{M}}{\bar{\gamma}_{s d}}\right) \\
C_{n}=\left[\frac{1}{1+g \bar{\gamma}_{s d}}\right]^{n} \exp \left(-\frac{\gamma_{M}}{\bar{\gamma}_{s d}}\right)
\end{gathered}
$$

[4] - X. Lagrange, "Throughput of HARQ protocols on a block fading channel," IEEE Communications Letters, vol. 14, pp. 257-259, March 2010. 


\section{Probability of error (3)}

- $P_{r d}\left(n, n^{\prime}\right)$ can be expressed as:

$$
\begin{gathered}
\operatorname{Prd}_{r d}\left(n, n^{\prime}\right)=\underbrace{\int_{0}^{\infty} \cdots \int_{0}^{\infty}}_{n^{\prime} \text { folds }} \underbrace{\int_{0}^{\infty} \cdots \int_{0}^{\infty} f\left(\gamma_{1}\right) f\left(\gamma_{1}+\gamma_{2}\right) \cdots}_{n-n^{\prime} \text { folds }} \\
\begin{array}{c}
f\left(\gamma_{1}+\gamma_{2}+\ldots \gamma_{n}\right) \times p_{\gamma_{s}}\left(\gamma_{1}\right) \cdots p_{\gamma_{s}}\left(\gamma_{n^{\prime}}\right) \\
p_{\gamma_{r}}\left(\gamma_{n^{\prime}+1}\right) \cdots p_{\gamma_{r}}\left(\gamma_{n}\right) d \gamma_{1} d \gamma_{2} \ldots d \gamma_{n}
\end{array}
\end{gathered}
$$

- $P_{r d}\left(n, n^{\prime}\right)$ can be approximated by:

$$
\begin{aligned}
P_{r d}\left(n, n^{\prime}\right) & \approx \underbrace{\int_{0}^{\infty} \cdots \int_{0}^{\infty}}_{n^{\prime} \text { folds }} \underbrace{\int_{0}^{\infty} \cdots \int_{0}^{\infty}}_{n-n^{\prime} \text { folds }} f\left(\gamma_{1}+\gamma_{2}+\ldots+\gamma_{n}\right) \\
& \times p_{\gamma_{s}}\left(\gamma_{1}\right) \cdots p_{\gamma_{s}}\left(\gamma_{n^{\prime}}\right) p_{\gamma_{r}}\left(\gamma_{n^{\prime}+1}\right) \cdots p_{\gamma_{r}}\left(\gamma_{n}\right) \\
& d \gamma_{1} d \gamma_{2} \ldots d \gamma_{n}
\end{aligned}
$$




\section{Simulation results: System model parameters}

\begin{tabular}{ccc}
\hline \hline Parameters & Description & value \\
\hline$P_{\text {txElec }}$ & transmitter circuitry power & $20 \mathrm{dBm}$ \\
$N_{0}$ & power spectral density of the noise & $-155 \mathrm{dBm} / \mathrm{Hz}$ \\
$N_{b}$ & number of bits in data packet & 1080 \\
$R_{b}$ & transmission bit rate & $150 \mathrm{kbps}$ \\
$R_{C}$ & coding rate & 1 \\
$P_{r \times E l e c}$ & receiver circuitry power & $20 \mathrm{dBm}$ \\
$f_{C}$ & carrier frequency & $2.4 \mathrm{GHz}$ \\
$\tau$ & ACK ratio & 0.08125 \\
$a$ & curve fitting parameter & 67.7328 \\
$g$ & curve fitting parameter & 0.9819 \\
$\gamma_{M}$ & SNR fitting parameter & $6.3281 \mathrm{~dB}$ \\
\hline
\end{tabular}

Table: System model parameters [5-6]

[5]- Q. Liu, S. Zhou, and G. Giannakis, "Cross-Layer combining of adaptive modulation and coding with truncated ARQ over wireless links, "IEEE Transactions on Wireless Communications, vol. 3, pp. 1746-1755, September 2004.

[6]- Z. Ruifeng, J.-M. Gorce, and K. Jaffres-Runser, "Low Bound of Energy- Latency Trade-Off of Opportunistic Routing in Multi-Hop Networks,"' in ICC'09. IEEE International Conference on Communications, 2009, pp. 1-6, June 2009. 


\section{Simulation results: PER versus effective SNR}

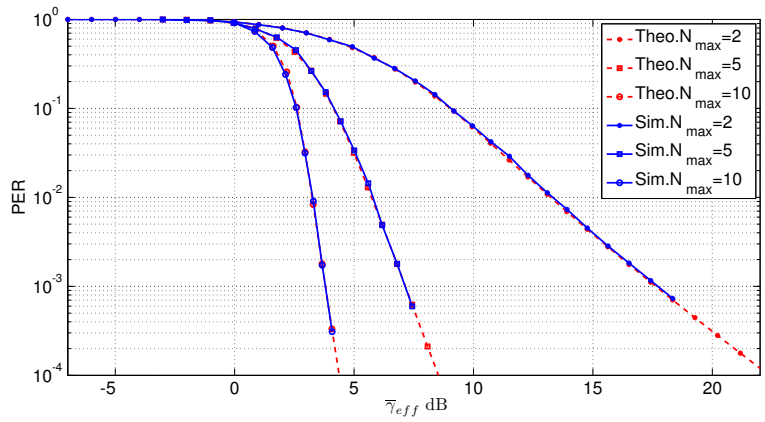

- The average effective SNR is defined as $\bar{\gamma}_{\text {eff }}=\bar{\gamma}_{s d} \cdot \bar{N}_{t_{c o o p}}$

- The angle between the line joining BS to $D$ and $B S$ to $R S$ is $\pi / 6$ radians

- $N_{\max }=2,5$ and 10

- Propagation model: COST 231-Hata $139.90+34.41 . \log _{10}\left(d_{l}\right) \mathrm{dB}$ in each link 


\section{Simulation results: Average delay versus $P_{t}$}

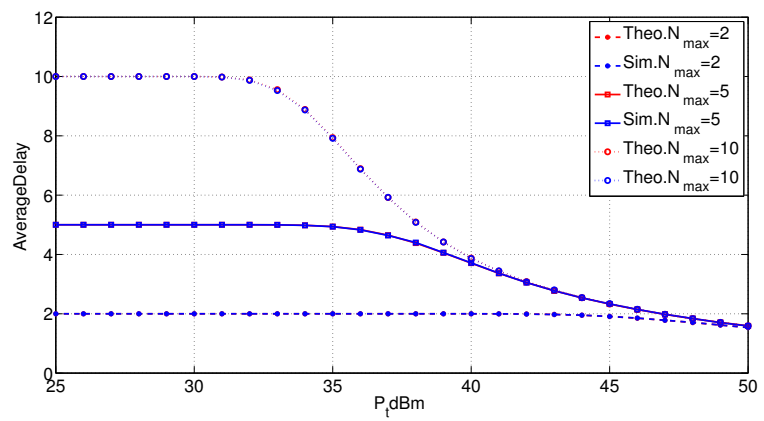

- $N_{\max }=2,5$ and 10

- The theoretical approximation matches perfectly the simulated one 


\section{Simulation results: Energy efficiency versus $P_{t}$}

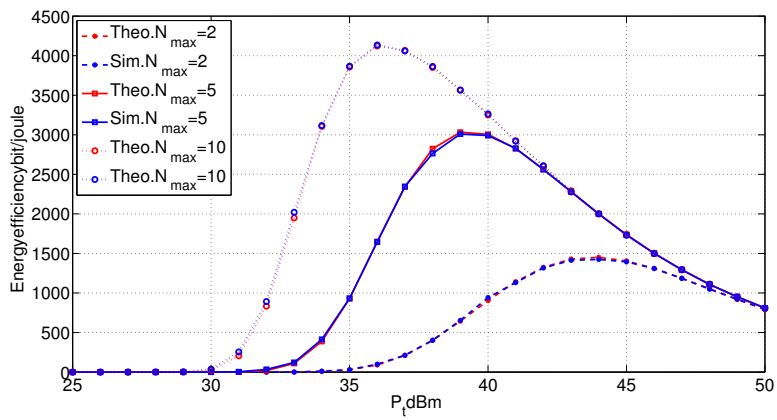

- As $N_{\max }$ increases, $\eta_{E}$ increases

- There is an optimal power that maximizes $\eta_{E}$ 


\section{Simulation results: Energy efficiency versus distance}

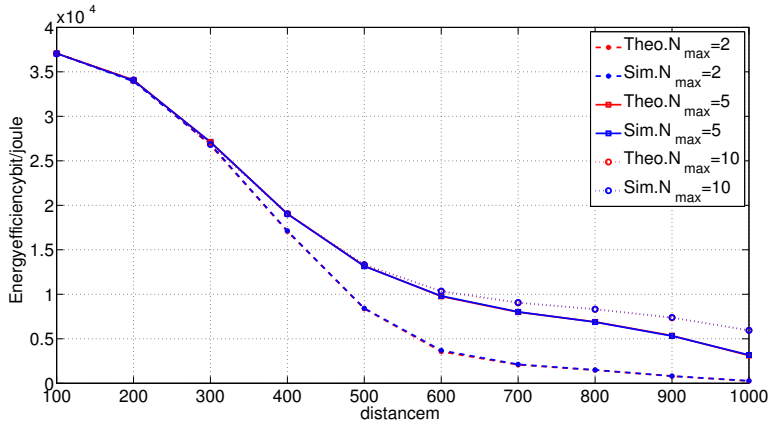

- $P_{t}=35 \mathrm{dBm}$

- BS, RS and D are collinear

- Higher number of retransmissions is energy efficient 


\section{Conclusions and future works}

\section{Conclusions}

- A simple theoretical analysis for energy efficiency in relay network

- Higher number of allowed retransmissions leads to higher energy efficiency communications

- Gain is assessed precisely with our model

\section{Future works}

- Resource allocation for OFDMA relay-assisted system for delay constrained users is under investigation

- This resource allocation takes into account the random distribution of users, the retransmission mechanisms and the coding scheme (LDPC) 